\title{
Determination of the dynamic stress intensity factor of a specimen under one-point bending from the measurement of the load-point displacement
}

\author{
J.A. Loya ${ }^{1}$, I. Villa ${ }^{1}$, J. Fernández-Sáez ${ }^{1}$ and C. Navarro ${ }^{1}$ \\ ${ }^{1}$ Department of Continuum Mechanics and Structural Analysis, \\ University Carlos III of Madrid, 28911 Leganés, Madrid, Spain
}

\begin{abstract}
The use of dynamic one-point bending tests as an alternative to three-point bending tests allows fracture properties such as dynamic initiation fracture toughness to be obtained at high strain rate. To perform this kind of test, experimental devices based on modifications of the Hopkinson bar can be used. Several authors have been proposed simplified procedures to obtain the dynamic stress intensity factor, $K_{I}(t)$, considering the specimen as a Timoshenko cracked beam subjected to a concentrated load at the central cross-section. The disadvantage of this procedure is that normally it is difficult to measure the force applied to the specimen in the above-mentioned tests. Here a simplified method is proposed for the calculation of $K_{I}(t)$, based on an analysis of the behaviour of a Timoshenko cracked beam, knowing the displacement of the point of loading, which can be measured more accurately than the applied load. The results were compared with those of a finite element numerical simulation and good agreement was found.
\end{abstract}

\section{INTRODUCTION}

One-point dynamic bending tests may be used instead of three-point tests to determine the initial fracture toughness, $K_{I d}$. This type of test was used by Giovanola [1,2]. A mobile mass is made to impact a notched specimen as in a three-point bending test, but with the important difference that the specimen is not supported, so the stress and deformations produced in the specimen are due only to the inertial forces. If, as a consequence of the loads generated, critical conditions are reached at the end of the notch, it will propagate. The impacting mass may be the striker of a Charpy pendulum or a mass projected by a pneumatic device. When the fracture properties of a material must be obtained at high strain rate, the one-point bending test may be performed using experimental devices based on modifications of the Hopkinson bar [3-5]. These systems consist of a bar of a given length and diameter (striker bar) which is put in contact with the central section of a cracked specimen identical to that which would be used in a three-point bending test. The bar is impacted by a shorter bar of the same diameter (projectile), generating a compressive pulse that travels along the bar. When it reaches the specimen, one part is transmitted to the specimen and another part is reflected back along the striker as a tensile load. Both the deformation caused by the compressive projectile, $\varepsilon_{i}(t)$, and that generated by the reflected load, $\varepsilon_{r}(t)$, are measured by strain gages attached to the striker bar. From these measurements it is possible to calculate the load applied to the specimen, $F(t)$, and also the displacement of the end of the bar initially in contact with the specimen, $u_{p}(t)$, by means of:

$$
F(t)=A_{b} E_{b}\left(\varepsilon_{i}(t)+\varepsilon_{r}(t)\right) \text { and } u_{p}(t)=c_{0} \int_{0}^{t}\left(\varepsilon_{i}(\tau)-\varepsilon_{r}(\tau)\right) d \tau
$$

in which $A_{b}$ stands for the area of the circular section of the bar, $E_{b}$ for its Young modulus and $c_{o}$ is the propagation speed of longitudinal waves along the material of the bar.

To obtain $K_{l}(\mathrm{t})$ in one-point bending tests, the Finite Element method may be used [3-7], but other simplified methods have been used. These methods are based on the modal analysis of a cracked beam subjected to a known load in its central section. With this procedure, the displacement of any section 
of the beam can be calculated. The stress intensity factor, $K_{I}(t)$, is assumed to be proportional to the difference between the displacement of the point of loading and the displacement of the point that would have been supported in a three -point bending test. The constants of proportionality adopted are those of three-point bending in static conditions. This type of analysis was made by Kishimoto et al. [8], considering the specimen as an Euler-Bernoulli beam. However, the geometry of the test beams, of span to width ratio equal to 4 , indicates that the specimen must be considered as a cracked Timoshenko beam [9].

The drawback of this method, as confirmed by several authors $[4,7]$ is that the force is not easily measured accurately in a Hopkinson bar, at least when the strains are measured in only one section of the striker bar.

We propose here a simplified method of calculating $K_{I}(t)$, based on the analysis of the behaviour of a cracked Timoshenko beam, knowing the loading point displacement, a variable that can be measured more easily than can the applied load.

\section{PROBLEM FORMULATION AND ITS SOLUTION}

We consider a Timoshenko beam of length $L$, width $W$ and thickness $B$, with a crack of length $a$ at its central section. The beam is without support and is subjected to a dynamic load $F(t)$ concentrated in its central section (see Fig. 1).

The beam is considered a one-dimensional solid, and using the modal superposition method, the temporal evolution of the vertical displacements of the sections, $y(\xi, t)$ and of their bending slopes, $\Psi(\xi, t)$, may be expressed as :

$$
y(\xi, t)=\sum_{i=1}^{\infty} Y_{i}(\xi) q_{i}(t)+Y_{T}(\xi) q_{T}(t) \text { and } \Psi(\xi, t)=\sum_{i=1}^{\infty} \varphi_{i}(\xi) q_{i}(t)
$$

in which $\xi$ is the dimensionless variable with respect to the length $L$ that stands for the position of any section of the beam. Functions $Y_{i}(\xi)$ and $\varphi_{i}(\xi)$ are the modes of vibration associated with displacements, $y(\xi, t)$ and bending slopes, $\Psi(\xi, t)$, and $Y_{T}(\xi)$ is the mode associated with the movement of the specimen as a rigid solid, which can be taken in general as equal to unity. The functions $q_{i}(t)$ and $q_{T}(t)$ are obtained as solutions to the following second-order differential equations:

$$
\ddot{q}_{i}+\omega_{i}^{2} q_{i}=\frac{F(t) Y_{i}\left(\frac{1}{2}\right)}{m_{i}} \text { and } q_{T}=\frac{F(t)}{m_{p}}
$$

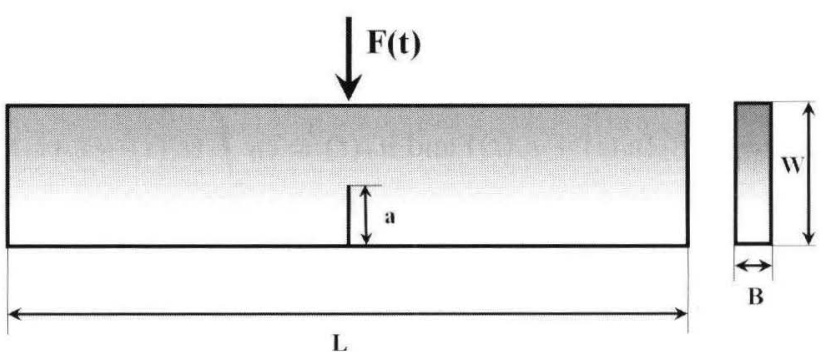

Figure 1. Geometry of the test specimen. 
$\omega_{i}$ are the vibration frequencies of the specimen, $m_{p}$ its total mass, and $m_{i}$ the masses associated with each mode of vibration, given by:

$$
m_{i}=2 \rho A L \int_{0}^{\frac{1}{2}}\left(Y_{i}^{2}(\xi)+\frac{I}{A} \varphi_{i}^{2}(\xi)\right) d \xi
$$

in which $\rho, A$ and $I$ are respectively the density, the area of the cross-section of the beam, and the moment of inertia of this section. The expressions for the frequencies, $\omega_{i}$, and for the modes of vibration, $Y_{i}(\xi)$ and $\varphi_{i}(\xi)$ of a Timoshenko beam with a crack in the central section and without support (free-free cracked beam) are given in [9]. Taking the initial conditions $q_{i}(0)=q_{T}(0)=0$ and $\dot{q}_{i}(0)=\dot{q}_{T}(0)=0$, the solutions of equation (5) and of (6), are obtained as:

$$
\begin{gathered}
q_{i}(t)=\frac{Y_{i}\left(\frac{1}{2}\right)}{m_{i} \omega_{i}} \int_{0}^{t} F(\tau) \sin \omega_{i}(t-\tau) d \tau \\
q_{T}(t)=\int_{0}^{t}\left(\int_{0}^{\eta} \frac{F(\tau)}{m_{p}} d \tau\right) d \eta
\end{gathered}
$$

Then, from the variables $q_{i}(t)$, the stress intensity factor, $K_{I}(t)$, can be written as:

$$
K_{I}(t)=\frac{3}{2} \frac{L}{B W^{3 / 2}} \frac{f(a / W)}{C} \sum_{i=1}^{n}\left(Y_{i}\left(\frac{1}{2}\right)-Y_{i}(0)\right) q_{i}(t)
$$

in which $n$ is the number of vibration modes considered and the function $f(a / W)$ for a specimen with $L / W=4$ is given by [10]:

$$
f\left(a_{/ W}\right)=\frac{\sqrt{a / W}}{(1-a / W)^{3 / 2}(1+a / W)}\left(1.90+0.41 a / W+0.51(a / W)^{2}-0.17(a / W)^{3}\right)
$$

$C$ is the compliance of the three-point bend specimen whose expression is given in [10]. In the test performed with experimental devices based on modifications of the Hopkinson bar, the loading point displacement can be measured more easily than can the applied load. Then, if the displacement of the loading point, $u_{p}(t)$, is known, considering only the first mode of vibration we have:

$$
u_{p}=y\left(\frac{1}{2}, t\right)=Y_{1}\left(\frac{1}{2}\right) q_{1}(t)+q_{T}(t)
$$

The previous equation can be combined with eq. (5), (with $i=1$ ) and with eq. (6) to obtain:

$$
\left(1+\frac{m_{p}}{m_{1}} Y_{1}^{2}\left(\frac{1}{2}\right)\right) \ddot{q}_{1}+\omega_{1}^{2} q_{1}=\frac{m_{p}}{m_{1}} Y_{1}\left(\frac{1}{2}\right) \ddot{u}_{p}
$$

Eq. (13) can be rewritten as:

$$
\ddot{q}_{1}+\omega_{d}^{2} q_{1}=k \ddot{u}_{p}
$$

where

$$
\omega_{d}=\frac{\omega_{1}}{\sqrt{1+\frac{m_{p}}{m_{1}} Y_{1}^{2}\left(\frac{1}{2}\right)}} \text { and } k=\frac{\frac{m_{p}}{m_{1}} Y_{1}\left(\frac{1}{2}\right)}{1+\frac{m_{p}}{m_{1}} Y_{1}^{2}\left(\frac{1}{2}\right)}
$$


Then, the solution of eq. (14) is given by:

$$
q_{1}(t)=\frac{k}{\omega_{d}} \int_{0}^{t} \ddot{u}_{p}(\tau) \sin \omega_{d}(t-\tau) d \tau
$$

The above expression relates the variable $q_{1}(t)$ to the second temporal derivative of the displacement of the loading point, $\ddot{u}_{p}(t)$.Taking into account the initial conditions, and with two integrations by parts, finally we obtain:

$$
q_{1}(t)=k u_{p}(t)-k \omega_{d} \int_{0}^{t} u_{p}(\tau) \sin \omega_{d}(t-\tau) d \tau
$$

The variable $q_{1}(t)$ is expressed now related to the displacement of the loading point, $u_{p}(t)$, and $K_{t}(t)$ is determined from eq. (10) taking $n=1$.

\section{NUMERICAL SIMULATION}

To check the validity of the simplified methods of determining $K_{t}(t)$, the results were compared with those obtained by the Finite Element method implemented in the commercial code ABAQUS [11].

A two-dimensional simulation was made of an aluminium specimen of $L=80 \mathrm{~mm}, W=20 \mathrm{~mm}$, $B=10 \mathrm{~mm}$ and $a=10 \mathrm{~mm}$. The Young modulus was $E=72 \mathrm{GPa}$, the Poisson coefficient $v=0.33$, and the material density $\rho=2800 \mathrm{~kg} / \mathrm{m}^{3}$. Given the symmetry of the problem, half the specimen was modelled, with 1180 four-nodes elements in plane deformation and with reduced integration, refining the mesh in the zone of the crack tip. Three different loading histories were considered (see Fig. 2.)

Load is rated with the maximum load $\left(F_{\max }=10 \mathrm{kN}\right)$ and the time with the oscillation period of the specimen corresponding to the fundamental frequency $(T=120 \mu \mathrm{s})$. The shapes of the waves are similar, with the same maximum load and the difference only in its duration. To integrate the equations of movement, the Hilber-Hughes-Taylor method was used [11]. A temporal increment of $1 \mu$ s was used in all the analyses. $K_{I}(t)$ was obtained from the dynamic $J$ integral, $J^{\prime}$, as proposed by Nishioka and Atluri [12] (see also [13]). For a stationary crack which is deformed in mode $I$, as in this case, $K_{I}(t)$ may be written as $[12,13]$ :

$$
K_{I}(t)=\sqrt{\frac{E J_{1}^{\prime}(t)}{1-v^{2}}}
$$

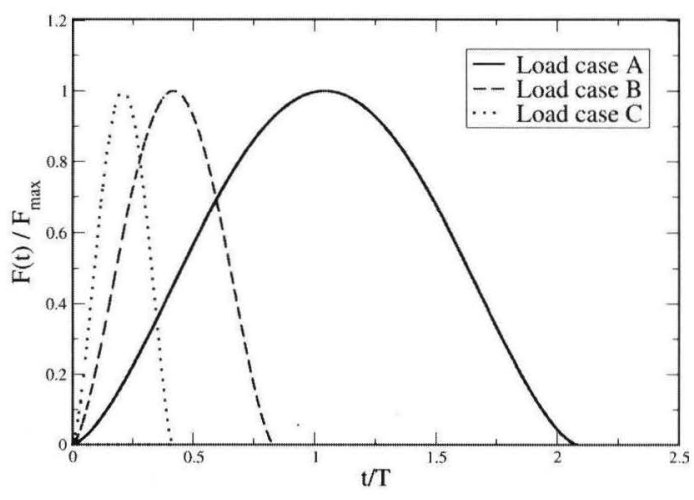

Figure 2. Loading histories considered. 


\section{RESULTS AND DISCUSSION}

In Figs. 3 to 5, a comparison of the values of $K_{I}(t)$ obtained by numerical simulation and those of the simplified methods, for the three loading conditions is shown. The stress intensity factor is given in dimensionless form, with respect to a reference value $\left(K_{0}=5.0 \mathrm{MPa} \mathrm{m}{ }^{1 / 2}\right)$ and the time in dimensionless form too, with respect to the value $T=120 \mu$ s as mentioned before.

The stress intensity factor obtained from the applied load shown in these figures was calculated using only the first mode of vibration. The consideration of higher modes does not affect the final results.

The tendencies observed in the results can be summarized as follows:

a) In Load case $\mathrm{A}$ in which the duration of the applied load is more or less twice that of the oscillation period of the cracked specimen, the results obtained by the simplified methods are very close to those of the Finite Element simulation.

b) When the duration of the applied loads is similar to the oscillation period of the cracked specimen (Load case B), there is a wider difference between the simplified results in the two methods analysed and between these and the numerical results. In the first instants of the loading process, the solution obtained from the applied load is almost coincident with the numerical one, but when the time (dimensionless) is above $t / T=0.5$, the solution obtained from the displacement of the loading point is closer to the numerical result.

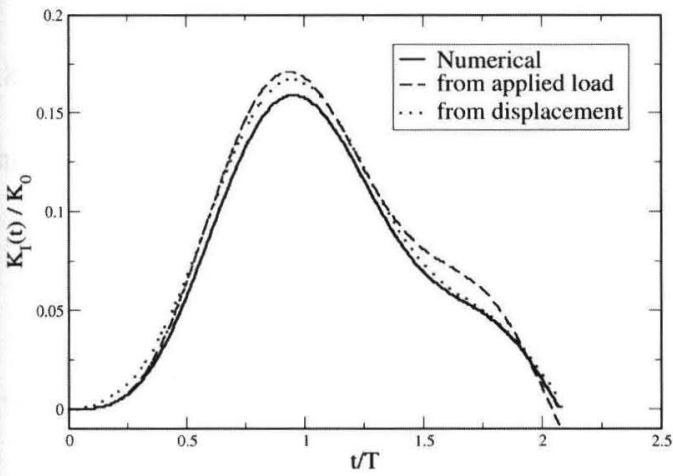

Figure 3. Temporal evolution of the stress intensity factor. Load case A.

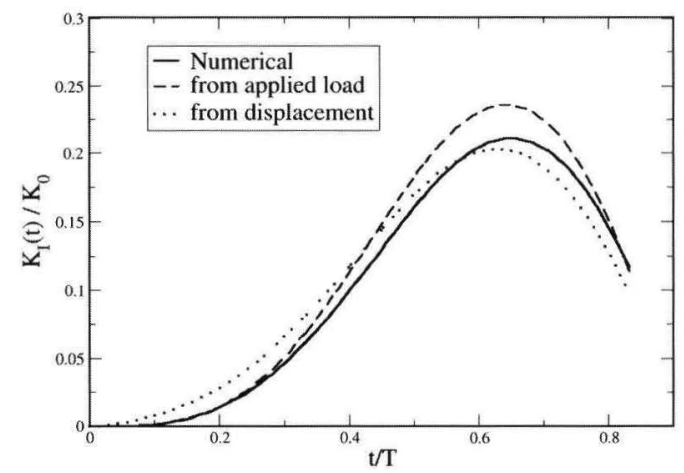

Figure 4. Temporal evolution of the stress intensity factor. Load case B.

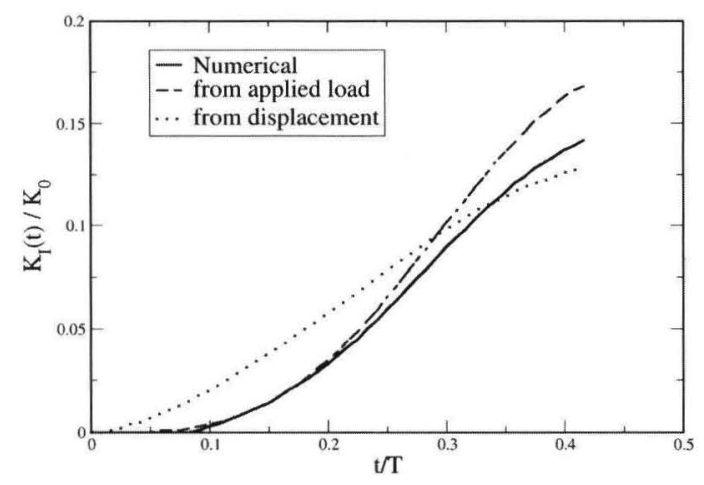

Figure 5. Temporal evolution of the stress intensity factor. Load case C. 
c) With the shortening of the duration of the pulse, the solutions based on the approximated methods differ more widely from the bidimensional numerical solution (load Case C). This may be because the analysis should include 2-D effects in frequencies and vibration modes that have not been considered in the present analysis.

\section{CONCLUSIONS}

For the measurement of the fracture properties of a material, at high-strain rate, one-point bending tests based on modifications of the Hopkinson bar may be used. To evaluate $K_{I}(t)$, numerical techniques such as Finite Elements can be used, as well as simplified procedures based on the analysis of the behaviour of a cracked Timoshenko beam subjected to a known load in its central section. The drawback in this case is that it is not always easy to measure accurately the load applied to the specimen. In this work we propose a simplified method for calculating $K_{I}(t)$ from the analysis of the behaviour of a cracked Timoshenko beam, knowing the loading point displacement. It is easier to measure accurately this latter variable than to measure the applied load.

The results of the simplified method agree well with those obtained numerically from twodimensional finite element simulations, when the loading lasts twice as long as the oscillation of the cracked beam. Differences arise with shorter load pulses, probably due to 2-D effects that have not been considered in the simplified procedures.

\section{References}

[1] J.H. Giovanola, "The one-point-bend impact test", Metals Handbook Mechanical Testing, Vol.8. American Society for Metals 271-276 (1985).

[2] J.H. Giovanola, "Investigation and application of the one-point-bend impact test", American Society for Testing and Materials. ASTM STP 905 307-328 (1986).

[3] H. Wada, M. Seika, C.A. Calder, T.C. Kennedy, Eng. Frac. Mech. 46 (1993) 715-719.

[4] G. Weisbrod, D. Rittel, Int. J. Fract. 104 (2000) 89-103.

[5] D. Rittel, A. Pineau, J. Clisson, L. Rota, Exp. Mech. 42 (2002) 1-6.

[6] L. Rubio, J. Fernández-Sáez, C. Navarro, Anales de Mecánica de la Fractura 15 (1998) 97-101.

[7] Rubio, L., "Determinación de parámetros de fractura dinámica a alta velocidad de deformación", Tesis Doctoral, Universidad Carlos III de Madrid, Madrid, España (1999).

[8] K. Kishimoto, Y. Fujino, S. Aoki, M. Sakata, JSME Int. J. 33 (1990) 51-56.

[9] P.R. Marur, Eng. Fract. Mech. 67 (2000) 41-53.

[10] G.V. Guinea, J.Y. Pastor, J. Planas and M. Elices, Int. J. Fract. 89 (1998) 103-116.

[11] ABAQUS User's Manual Version 5.8, Hibbitt, Karlsson \& Sorensen, Inc. (1997).

[12] T. Nishioka, S.N. Atluri, Eng. Fract. Mech. 18 (1983) 1-22.

[13] T. Nishioka, in FRACTURE: A Topical Encyclopaedia of Current Knowledge Dedicated to Alan Arnold Griffith, edited by G.P. Cherepanov (Krieger Publishing Company, Malabar, Florida, USA, 1998), 575-617. 